\title{
Observation of vortex nucleation in a rotating two-dimensional lattice of Bose-Einstein condensates.
}

\author{
R. A. Williams, S. Al-Assam, and C. J. Foot \\ Clarendon Laboratory, Department of Physics, University of Oxford, \\ Parks Road, Oxford, OX1 3PU, United Kingdom
}

(Dated: August 20, 2021)

\begin{abstract}
We report the observation of vortex nucleation in a rotating optical lattice. $\mathrm{A}{ }^{87} \mathrm{Rb}$ Bose-Einstein condensate was loaded into a static two-dimensional lattice and the rotation frequency of the lattice was then increased from zero. We studied how vortex nucleation depended on optical lattice depth and rotation frequency. For deep lattices above the chemical potential of the condensate we observed a linear dependence of the number of vortices created with the rotation frequency, even below the thermodynamic critical frequency required for vortex nucleation. At these lattice depths the system formed an array of Josephson-coupled condensates. The effective magnetic field produced by rotation introduced characteristic relative phases between neighbouring condensates, such that vortices were observed upon ramping down the lattice depth and recombining the condensates.

PACS numbers: $03.75 . \mathrm{Lm}, 67.85 . \mathrm{Hj}, 74.50 .+\mathrm{r}, 74.81 . \mathrm{Fa}$
\end{abstract}

Ultracold quantum gases in rotating optical lattices find themselves at the intersection of two fields which have generated an impressive body of experimental and theoretical work. The versatile and clean potentials offered by optical lattices have proved to be an incredibly adept system for exploring a wide range of fundamental problems in condensed matter physics such as the Mott-insulator transition [1, Anderson localization [2, 3] and Tonks-Girardeau gases [4. Similarly rotating quantum gases have provided an array of striking results, from the nucleation of vortex lattices in Bose gases 5, 6 to rotating Fermi gases at the BCS-BEC crossover [7. The close analogy between the physics of rapidly rotating neutral atoms and electrons under a magnetic field has led to considerable interest in the possibility of achieving strongly-correlated quantum Hall states in a rapidly rotating atomic gas [8, 9].

Rotating optical lattices for cold atoms have generated a large amount of interest in their own right, which can broadly be divided into three main areas: (i) Weak rotating lattices have been used to pin vortices [10] and theoretical work has shown that rich vortex lattices structures, including lattices of doubly quantized vortices, are predicted to emerge [11, 12]; (ii) Stronger optical lattice potentials with a large number of atoms per site (100-1000) realize the physics of Josephson junction arrays (JJA) under magnetic fields [13, 14]; (iii) Arguably the most interesting regime is for a dilute gas in the tight binding regime in a rotating lattice where fractional quantum Hall physics is predicted to occur 15-17. In all three regimes rich structures emerge that depend on the relative density of vortices and lattice sites.

We report on the first experiments with a rotating optical lattice in the 'deep' lattice regime where lattices depths were reached such that a $2 \mathrm{D}$ array of weakly linked condensates was created, forming a Josephson junction array. Under rotation this system realizes the uniformly frustrated JJA [13, 18. We have investigated the nucleation of vortices in a rotating lattice, starting from a nonrotating condensate loaded into a static lattice, and then increasing the rotation rate of the lattice. There has been one previous experiment employing a rotating optical lattice by Tung et al. [10] at JILA, where it was reported that heating due to mechanical instabilities and aberrations limited the rotating lattice to depths less than $30 \%$ of the condensate's chemical potential. We were not limited to this weak lattice regime and our system is markedly different to the JILA experiment where a vortex lattice was created by an elegant evaporative spin-up technique [6] before a weak co-rotating optical lattice was imposed.

The rotating lattice used in the experiments described in this paper was generated by dual-axis acousto-optic deflectors and a novel optical system, described in detail in 19. The two-dimensional optical lattice was formed in the focal plane of a custom-made multi-element lens with a numerical aperture of 0.27 . Generating the rotation of the lattice acousto-optically gave intrinsically smooth rotation and allowed the rotation rate to be varied during an experimental run.

The Coriolis force on neutral atoms in the rotating frame has the same form as the Lorentz force experienced by charged particles in a magnetic field. Rotation at angular frequency $\Omega$ gives rise to the effective magnetic vector potential $\mathbf{A}=\boldsymbol{\Omega} \times \mathbf{r}$, and the Peierls phase gained by an atom tunnelling from site $i$ to site $j$ is $A_{i j}=(m / \hbar) \int_{\mathbf{r}_{i}}^{\mathbf{r}_{j}} \mathbf{A}\left(\mathbf{r}^{\prime}\right) \cdot \mathrm{d} \mathbf{r}^{\prime}$, where $m$ is the mass of the atom. The sum of the link phases around a lattice plaquette of side $d$ obeys the property $\sum_{\text {plaquette }} A_{i j}=2 \pi f$, where $f=2 m \Omega d^{2} / h$ is the mean number of vortices per lattice plaquette.

The experiments started with a ${ }^{87} \mathrm{Rb}$ condensate containing $2 \times 10^{5}$ atoms with no visible thermal component, held in a trap formed by an axial symmetric Ioffe- 
Pritchard trap and a single red-detuned light sheet in the radial plane increasing the axial trapping frequency to give $\left\{\omega_{r}, \omega_{z}\right\}=2 \pi\{20.1,53.0\} \mathrm{Hz}$. The residual radial anisotropy of the combined magnetic and optical trap was $\omega_{y} / \omega_{x}=1.008 \pm 0.003$. The dipole sheet trap was creating using broadband light with a spectral width $\Delta \lambda \sim 3 \mathrm{~nm}$ centred on $865 \mathrm{~nm}$ in order that the light had a short coherence length. This ensured there were no interference effects, e.g. from multiple reflections at the vacuum cell wall, adding noise to the trapping potential. With narrow-band light it was observed such perturbations acted to spin down the cloud and caused irregularities in the filling of the lattice.

The $2 \mathrm{D}$ optical lattice was formed in the radial plane of the trapped condensate by four circularly polarized beams at $\lambda=830 \mathrm{~nm}$ intersecting in the focal plane of a high N.A. objective lens, achieving a lattice constant of $2 \mu \mathrm{m}$. One pair of beams was detuned by $10 \mathrm{MHz}$ to the other pair ensuring interference between the orthogonal optical lattices did not affect the atoms. The optical lattice was initially static as it was ramped to its final depth $V_{0}$ in the range $100 \mathrm{~Hz} \leq V_{0} \leq 4000 \mathrm{~Hz}$ [20. The radial Thomas-Fermi radius of the condensate was $16 \mu \mathrm{m}$, resulting in the filling of $\sim 16$ lattice sites across the diameter of the condensate and around 200 sites in all. The lattice enhanced interaction energy at the central sites rose above the chemical potential of the unperturbed condensate $(\mu \approx 500 \mathrm{~Hz})$ meaning an array of individual condensates was not formed until $V_{0} \sim 1000 \mathrm{~Hz}$. The central wells contained around 1500 atoms.

After the condensate had been loaded into the static optical lattice $\Omega$ was increased linearly from zero to its final value in $320 \mathrm{~ms}$, followed by a further $100 \mathrm{~ms}$ of rotation at the final value. While the lattice was still rotating the lattice depth was then ramped down to zero in $12 \mathrm{~ms}$, allowing the condensates to merge and converting phase differences around plaquettes to vortices. Previously Scherer et al 21] have investigated vortex formation by merging three independent condensates, and Schweikhard et al [22] have observed the thermal activation of vortices on a two-dimensional lattice of Josephsoncoupled BECs, however our experiment takes place in the rotating frame. Rotation gives rise to an additional phase $2 \pi f$ around a plaquette of the optical lattice, simulating the effect of a magnetic field on charged particles. After the optical lattice was ramped down the cloud was then immediately released from the magnetic and optical traps and destructively imaged after $20 \mathrm{~ms}$ time-of-flight expansion. We also repeated the sequence but holding the cloud after the lattice was ramped down for an additional $500 \mathrm{~ms}$, to allow any vortex-antivortex pairs to annihilate (such pairs could be created in a static lattice [22]). This generally improved visibility of vortices in the harmonic trap and we observed the same number of vortices in the system within experimental error. The uncertainty in the number of vortices observed for a given
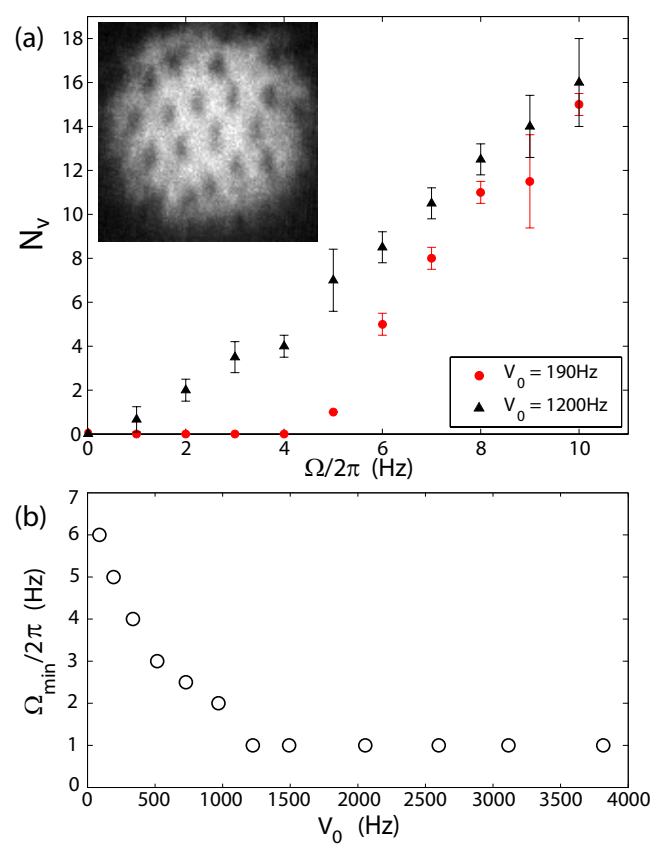

FIG. 1: (a) Number of vortices nucleated as a function of optical lattice rotation frequency $\Omega$ for two lattice depths, $V_{0}=190 \mathrm{~Hz}$ and $V_{0}=1200 \mathrm{~Hz}$. The error bars denote the standard deviation in vortex number for three experimental images. Inset: an example of a vortex lattice created by the rotating optical lattice of depth $V_{0}=1200 \mathrm{~Hz}$ at $\Omega$ / $2 \pi=10 \mathrm{~Hz}$. (b) The minimum rotation frequency needed to nucleate a single vortex, $\Omega_{\min }$, as a function of the lattice depth $V_{0}$ ( $1 \mathrm{~Hz}$ was the minimum frequency used).

$\Omega$ arose from difficulties in determining the presence of vortices near the edge of a condensate.

Figure 1(a) shows the number of vortices, $N_{v}$, observed as a function of the optical lattice rotation frequency, $\Omega$, for lattice depths of $V_{0}=190 \mathrm{~Hz}$ and $V_{0}=1200 \mathrm{~Hz}$. The behaviour of $N_{v}(\Omega)$ has a characteristic shape which depends on the exact vortex nucleation process. The first BEC stirring experiments used stirring beams of a similar size to the condensate and observed that no vortices were nucleated below $\Omega \approx 0.7 \omega_{r}$, where there is excitation of the $l=2$ surface mode 23 . It was later shown that sufficiently small stirring beams nucleate vortices without discrete resonances of surface modes [24, and the minimum frequency for vortex nucleation, $\Omega_{\min }$, matched the frequency at which a single vortex at the center of a condensate is energetically stable, $\Omega_{c}$ 25]. For our experimental conditions $\Omega_{c} / 2 \pi \approx 4 \mathrm{~Hz}$. For $V_{0}=190 \mathrm{~Hz}$, far below the $\approx 1000 \mathrm{~Hz}$ needed to split the condensate into weakly linked islands, Fig. 11(a) shows a similar trend to that observed in 24], i.e. $N_{v}$ did not exhibit sharp resonances and $\Omega_{\min } \approx \Omega_{c}$. We checked this behaviour was an equilibrium property by repeating the experiment with rotation times up to $1 \mathrm{~s}$, and observed the same response. We infer that a weak $\left(V_{0} \ll \mu\right)$ rotating lattice stirs up a condensate in a non-resonant fashion. For a 


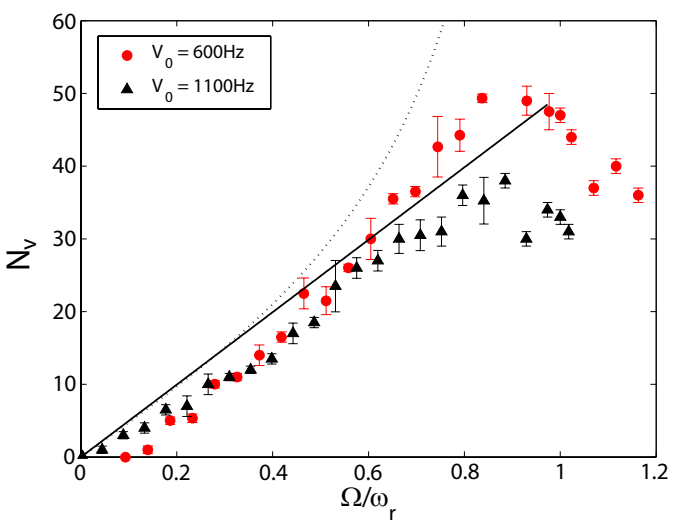

FIG. 2: Number of vortices nucleated as a function of $\Omega /$ $\omega_{r}$. The solid line is the number of vortices expected for a cloud of fixed radius, $R_{0}=16 \mu \mathrm{m}$, while the dashed line in the number of vortices expected for a centrifugally distorted cloud of radius $R=R_{0}\left[1-\left(\Omega / \omega_{r}\right)^{2}\right]^{-3 / 10}$. We observed the optical lattice prevented the cloud from reaching $R$ for a given $\Omega$, allowing rotation above the critical frequency $\Omega>\omega_{r}$ to be achieved.

rotating lattice of depth $V_{0}=1200 \mathrm{~Hz}$, however, $N_{v}$ displayed a distinctly different trend, with the number of vortices observed following a linear dependence on $\Omega$ even below $\Omega_{c}$. This change in behaviour is indicative of entering the regime where the system forms an array of Josephson-coupled condensates, rather than simply a condensate perturbed by a weak rotating lattice as for $V_{0}=190 \mathrm{~Hz}$. Figure 1(b) highlights the transition between the two regimes, showing the dependence of $\Omega_{\text {min }}$ on $V_{0} . \Omega_{\text {min }}$ falls below $\Omega_{c}$ as $V_{0}$ rises above $\mu=500 \mathrm{~Hz}$, and falls to $1 \mathrm{~Hz}$ (the minimum rotation frequency used) for $V_{0} \geq 1000 \mathrm{~Hz}$, corresponding to the lattice depth at which condensates are expected to be well localized on sites and can only communicate by tunneling. The rotation induced phase differences between neighbouring condensates are converted to vortices when the lattice is ramped down in the rotating frame. This nucleation mechanism creates vortices near the centre of the condensate even when $\Omega<\Omega_{c}$. Vortices do not have to spin in from the edge of the cloud, which is what we observed for stirring with a weak lattice potential.

The measurements of the number of vortices $N_{v}$ was extended to higher rotation frequencies $\Omega$ as shown in Figure 2. The ratio $\Omega / \omega_{r}$ is used where $\omega_{r}$ is the radial trapping frequency taking into account the slight enhancement by the Gaussian envelope of the lattice beams: for $V_{0}=600 \mathrm{~Hz}, \omega_{r} / 2 \pi=21.5 \mathrm{~Hz}$ and for $V_{0}=1100 \mathrm{~Hz}$, $\omega_{r} / 2 \pi=22.6 \mathrm{~Hz}$. The density of vortices for a given $\Omega$, whether a lattice is present or not, is $n_{v}=m \Omega / \pi \hbar$. The total number of vortices is then $N_{v}=m \Omega R^{2} / \hbar$. For a rotating BEC in equilibrium at $\Omega$ the centrifugal distortion of the Thomas-Fermi radius is $R=R_{0}\left[1-\left(\Omega / \omega_{r}\right)^{2}\right]^{-3 / 10}$ [26]. The predicted number of vortices for a cloud displaying this behaviour is denoted by the dashed line in

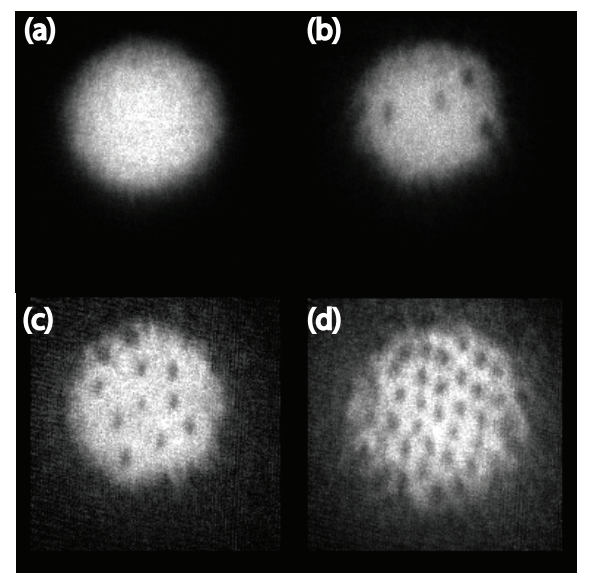

FIG. 3: Images after release from the rotating optical lattice showing the pattern of vortices: (a) Condensate before rotation; (b) $\Omega / 2 \pi=4 \mathrm{~Hz}$; (c) $\Omega / 2 \pi=7 \mathrm{~Hz}$; (d) $\Omega / 2 \pi=17 \mathrm{~Hz}$.

Fig. 2. The solid line presumes the radius stays constant at $R_{0}=16 \mu \mathrm{m}$. For $V_{0}=1100 \mathrm{~Hz}, N_{v}$ rises linearly with $\Omega$, whereas for the weaker $V_{0}=600 \mathrm{~Hz}$ lattice $N_{v}$ shows a faster, nonlinear dependence on $\Omega$. We attribute this behaviour to the deeper lattice suppressing the effect of centrifugal distortion. For the deeper lattice atoms may only redistribute themselves by tunneling between sites. While the tunneling parameter (the Josephson-coupling energy [13) is around $2 \mathrm{kHz}$ at central lattice sites this drops to $<50 \mathrm{~Hz}$ at peripheral lattice sites due to the drop in atom number, giving tunneling times at the edge of the lattice comparable to the experimental duration. Bloch oscillations may also play a role in inhibiting the atoms from spreading out in the lattice [27.

As a result of the optical lattice inhibiting the spreading out of the cloud we were able to rotate the cloud above the critical frequency $\Omega>\omega_{r}$. As seen in Fig. 2 the number of vortices dropped for rotation above $\omega_{r}$, due to increased heating of the condensate. However vortices could be observed up to $\Omega=1.15 \omega_{r}$ for $V_{0}=600 \mathrm{~Hz}$ before heating and reduction of vortex visibility meant it was no longer possible to observe vortices. The heating at $\Omega \sim \omega_{r}$ was worse for the deeper $V_{0}=1100 \mathrm{~Hz}$ lattice and vortices could not be observed above $\Omega=1.02 \omega_{r}$. A cloud in a harmonic trap without the presence of an optical lattice should be expelled for $\Omega>\omega_{r}$ though Bretin et al. were able to achieve $\Omega=1.06 \omega_{r}$ using additional quartic confinement [28].

The structure of the vortex patterns formed in a bosonic JJA under rotation are heavily influenced by the presence of the lattice. For values of the frustration parameter (the mean number of vortices per lattice plaquette) $f=2 m \Omega d^{2} / h=p / q$ (where $\mathrm{p}$ and q are integers) the ground state vortex patterns have been calculated [13, and have a unit cell of size $q \times q$ sites. Between these rational fraction values of $f$ intermediate patterns are formed. Figure 3 shows examples of the vortex patterns 


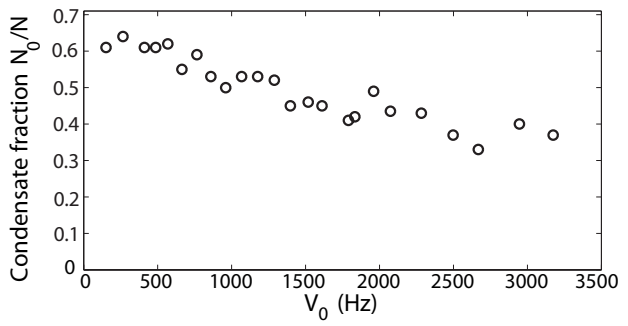

FIG. 4: Condensate fraction $N_{0} / N$ as a function of lattice depth $V_{0}$ after ramping up the rotation rate to $15 \mathrm{~Hz}$ in $40 \mathrm{~ms}$ followed by $35 \mathrm{~ms}$ of rotation at $15 \mathrm{~Hz}$.

we observe. We do not reproducibly observe the predicted $q \times q$ unit cell structure as we scan $\Omega$ and hence $f$. There are several possible reasons for this: (i) for $d=2 \mu \mathrm{m}$ and $\omega_{r} / 2 \pi=20.1 \mathrm{~Hz}$ the maximum value of $f$ we can reach is $f \sim 1 / 5$. The finite size of the system then becomes an issue, as it will be difficult to realize the periodic structure for $q=5$ when a maximum of 200 sites are filled by the condensate; (ii) the energy landscape of cold atoms in a rotating lattice is known to be very glassy, that is closely spaced minima in energy can be separated by large barriers, making it hard to reach the true ground state [29]. Lack of equilibration on experimental timescales might then account for the absence of $q \times q$ vortex structures; (iii) any intrinsic heating from the rotating optical lattice will make it difficult to reach the ground state.

The issue of finite size can be addressed by increasing $\omega_{z}$ or using a larger condensate, enabling more lattice sites to be filled. The values of $f$ achievable can be increased by implementing tighter radial trapping to allow higher $\Omega$ to be reached or increasing the lattice constant d. The $f=1 / 2$ case is particularly interesting due to competition between an Ising-type and BKT type phase transition 14. Vortex patterns in a bosonic JJA under rotation for $0 \leq f \leq 1 / 2$ will be explored in future work.

An important question regarding the viability of future rotating optical lattice experiments is the issue of heating. Figure 4 displays the fraction of atoms in the condensate, $N_{0} / N$, after rotation at $\Omega / 2 \pi=15 \mathrm{~Hz}(\Omega /$ $2 \pi$ was increased from 0 to $15 \mathrm{~Hz}$ in $40 \mathrm{~ms}$ followed by $35 \mathrm{~ms}$ of rotation at $15 \mathrm{~Hz}$ ) for different lattice depths, showing an approximately linear dependence. We found at $\Omega / 2 \pi=15 \mathrm{~Hz}, V_{0}=1100 \mathrm{~Hz}$ we could rotate for around $300 \mathrm{~ms}$ before heating significantly reduced the condensate fraction, with longer rotation times achievable for smaller $\Omega$ or $V_{0}$ or both. The main sources of heating were fluctuations in the lattice depth during rotation (at the $\pm 1 \%$ level) and residual imperfections in the lattice upon rotation. All the data in this paper were taken without using any additional evaporation during or after the rotation process (unlike previous stirring experiments [5, 23]). The implementation of an additional cool- ing scheme in the optical lattice such as that described by Griessner et al. 30] could enable sufficiently low temperatures to be achieved to realise quantum Hall physics in future experiments with the rotating lattice [15 17].

In conclusion, the use of acousto-optic deflection to generate smooth rotation of an optical lattice at a welldefined frequency has allowed us to explore a new method of vortex nucleation. For lattice depths $V_{0}<\mu$ we found the weak lattice acted as a stirring mechanism. For deeper lattices the system was split into well-localized condensates at each site and a linear dependence of vortex number on $\Omega$ was observed for rotation frequencies below the thermodynamic critical frequency for vortex nucleation. This implied vortices were created locally at a lattice plaquette.

This research is supported by EPSRC, QIP IRC (GR/S82176/01) and ESF.

[1] M. Greiner et al., Nature 415, 39 (2002).

[2] J. Billy et al., Nature 453, 891 (2008).

[3] G. Roati et al., Nature 453, 895 (2008).

[4] T. Kinoshita et al., Science 305, 1125 (2004).

[5] Abo-Shaeer et al., Science 292, 476 (2001).

[6] V. Schweikhard et al., Phys. Rev. Lett. 92, 040404 (2004)

[7] M. W. Zwierlein et al., Nature 435, 1047 (2005).

[8] N. K. Wilkin and J. M. F. Gunn, Phys. Rev. Lett. 84, 6 (2000).

[9] N. R. Cooper et al., Phys. Rev. Lett. 87, 120405 (2001).

[10] S. Tung et al., Phys. Rev. Lett. 97, 240402 (2006).

[11] K. Kasamatsu and M. Tsubota, Phys. Rev. Lett. 97, 240404 (2006).

[12] H. Pu et al., Phys. Rev. Lett. 94, 190401 (2005).

[13] K. Kasamatsu, Phys. Rev. A 79, 021604 (2009).

[14] M. Polini et al., Phys. Rev. Lett. 95, 010401 (2005).

[15] R. Bhat et al., Phys. Rev. A 76, 043601 (2007).

[16] R. N. Palmer and D. Jaksch, Phys. Rev. Lett. 96, 180407 (2006).

[17] A. S. Sørensen et al., Phys. Rev. Lett. 94, 086803 (2005).

[18] N. R. Cooper, Advances in Physics 57, 539 (2008).

[19] R. A. Williams et al., Opt. Express 16, 16977 (2008).

[20] $V_{0}$ was calibrated by Kapitza-Dirac diffraction with an uncertainty of $\pm 10 \%$. The measured $V_{0}$ agreed with that calculated from measured parameters within this error.

[21] D. R. Scherer et al., Phys. Rev. Lett. 98, 110402 (2007).

[22] V. Schweikhard et al., Phys. Rev. Lett. 99, 030401 (2007)

[23] K. W. Madison et al., Phys. Rev. Lett. 84, 806 (2000).

[24] C. Raman et al., Phys. Rev. Lett. 87, 210402 (2001).

[25] A. L. Fetter and A. A. Svidzinsky, J. Phys. C 13, R135 (2001).

[26] A. L. Fetter, Rev. Mod. Phys. 81, 647 (2009).

[27] T. Wang and S. Yelin, arXiv:quant-ph/0610114v3.

[28] V. Bretin et al., Phys. Rev. Lett. 92, 050403 (2004).

[29] D. S. Goldbaum and E. J. Mueller, Phys. Rev. A 79, 063625 (2009).

[30] A. Griessner et al., Phys. Rev. Lett. 97, 220403 (2006). 